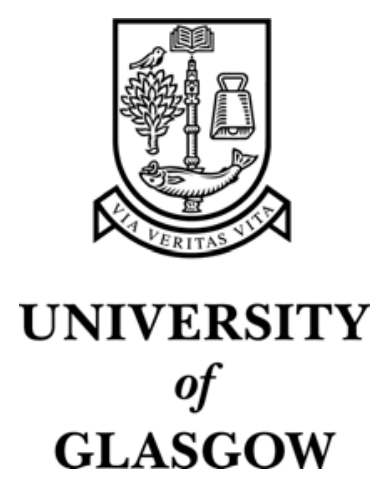

Paul, M. (2006) Natural convection flow from an isothermal horizontal cylinder in presence of heat generation. International Journal of Engineering Science 44(13-14):pp. 949-958.

http://eprints.gla.ac.uk/3326/ 


\title{
Natural Convection Flow from an Isothermal Horizontal Circular Cylinder in Presence of Heat Generation
}

\author{
Md. Mamun Molla ${ }^{1}$, Md. Anwar Hossain ${ }^{2}$, Manosh C Paul ${ }^{3 *}$ \\ ${ }^{1}$ Department of Mathematics, Bangladesh University of Engineering \& Technology, Bangladesh \\ ${ }^{2}$ Department of Mathematics, University of Dhaka, Dhaka-1000, Bangladesh \\ ${ }^{3}$ Department of Mechanical Engineering, University of Glasgow, Glasgow G12 8QQ, UK
}

\begin{abstract}
Natural convection laminar boundary layer flow from a horizontal circular cylinder with a uniform surface temperature at presence of heat generation has been investigated. The governing boundary layer equations are transformed into a non-dimensional form and the resulting nonlinear systems of partial differential equations are solved numerically applying two distinct methods namely (i) Implicit finite difference method together with the Keller box scheme and (ii) Series solution technique. The results of the surface shear stress in terms of the local skin friction and the surface rate of heat transfer in terms of the local Nusselt number for a selection of the heat generation parameter $\gamma(=$ $0.0,0.20 .5,0.8,1.0)$ are obtained and presented in both tabular and graphical formats. Without effect of the internal heat generation inside the fluid domain for which we take $\gamma$ $=0.0$, the present numerical results show an excellent agreement with those of Merkin [1]. The effects of $\gamma$ on the fluid velocity, temperature distribution, streamlines and isotherms are examined.
\end{abstract}

Keywords: Natural convection, Keller box, Heat generation, Heat transfer, Horizontal circular cylinder

\section{Nomenclature}

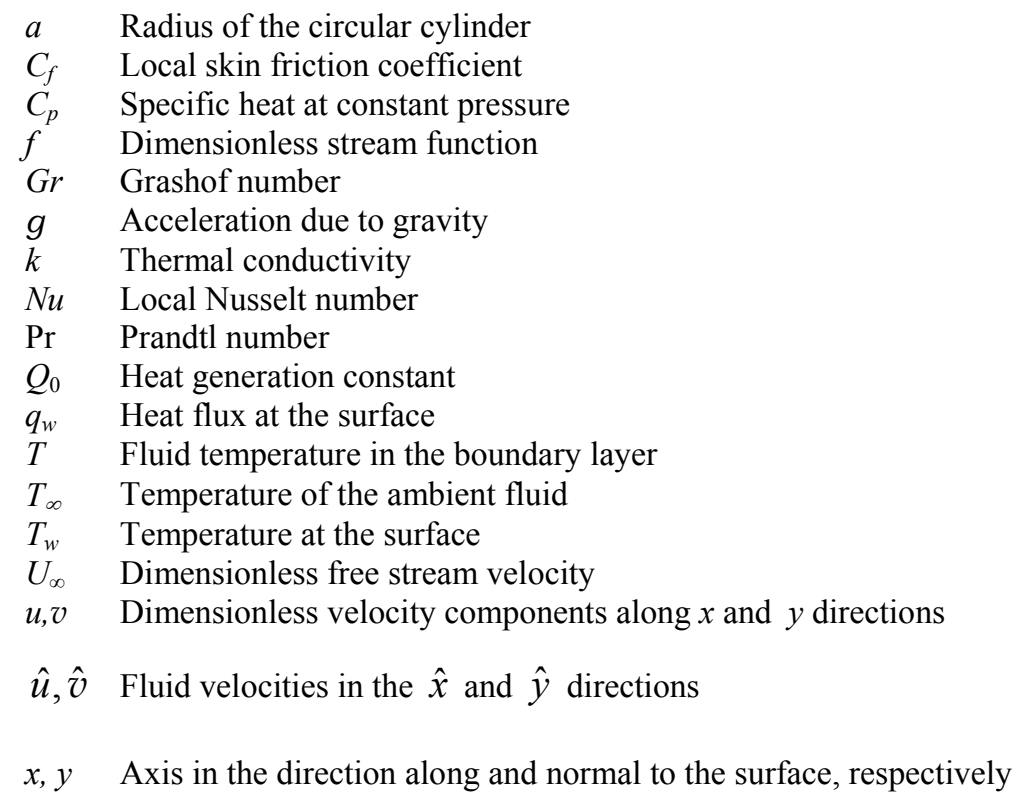

* Corresponding author: E-mail: m.paul@mech.gla.ac.uk, Tel: +44 (0)141 330 8466, Fax: +44 (0)141330 4343 . 


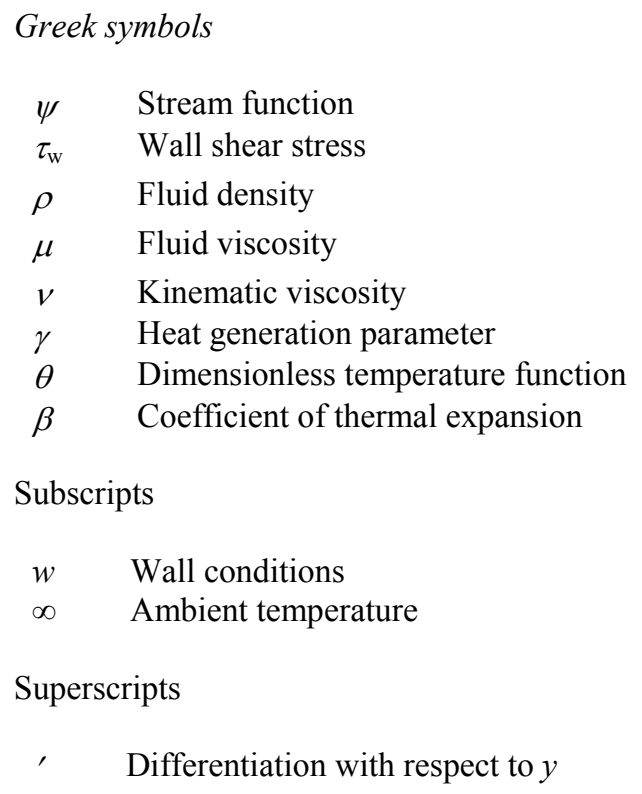

\section{Introduction}

A large number of physical phenomena involve natural convection driven by heat generation. The study of heat generation in moving fluids is important in view of several physical problems such as those dealing with chemical reactions and those concerned with dissociating fluids. Possible heat generation effects may alter the temperature distribution and, therefore, the particle deposition rate. This may occur in such applications related to nuclear reactor cores, fire and combustion modelling, electronic chips and semiconductor wafers. In fact, the literature is replete with examples dealing with the heat transfer in laminar flow of viscous fluids.

Vajravelu and Hadjinolaou [2] studied the heat transfer characteristics in a laminar boundary layer flow of a viscous fluid over a linearly stretching continuous surface with viscous dissipation/frictional heating and internal heat generation. In this study, they considered the volumetric rate of heat generation, $q^{\prime \prime \prime}\left[\mathrm{W} / \mathrm{m}^{3}\right]$, as

$$
q^{\prime \prime \prime}= \begin{cases}Q_{0}\left(T-T_{\infty}\right), & \text { for } T \geq T_{\infty} \\ 0, & \text { for } T<T_{\infty}\end{cases}
$$

where $Q_{0}$ is the heat generation constant. The above relation is valid for the state of some exothermic processes having $T_{\infty}$ as the onset temperature.

Effect of the heat generation or absorption and thermophoresis on a hydromagnetic flow with heat and mass transfer over a flat plate was investigated by Chamkha and Issa [3]. Also the effects of the conjugate conduction-natural convection heat transfer along a thin vertical plate with non-uniform heat generation have been studied by Mendez and Trevino [4]. Recently Molla et al. [5] have investigated the natural convection flow with heat generation/absorption along a uniform heated vertical wavy surface.

In 1976, Sparrow and Lee [6] looked at the problem of mixed convection over a heated horizontal circular cylinder. They obtained a solution by expanding the fluid ve- 
locity and temperature profiles in powers of $x$, the co-ordinate measuring distance from the lowest point on the cylinder. It appears that Merkin [1, 7] was the first author who presented a complete solution of this problem using the Blasius and Gortler series expansion methods along with an integral method and a finite-difference scheme. The problem of free convection boundary layer flow from cylinders of elliptic cross-section was also studied by Merkin [8].

Later Ingham [9] investigated the boundary layer flow on an isothermal horizontal cylinder. Recently Hossain and Alim [10] have studied the interaction of radiation with natural convection boundary layer flow along a vertical thin cylinder. Hossain et al. [11] have also studied radiation-conduction interaction on mixed convection from a horizontal circular cylinder. Very recently, natural convection flow from an isothermal horizontal circular cylinder with temperature dependent viscosity has been investigated by Molla et al. [12].

In micropolar fluid application, there appear to have some recent works published in the literature. For example see Pop et al. $[13,14]$, where they have considered the problem of natural convection flow from a lower stagnation point to an upper stagnation point of a horizontal circular cylinder immersed in a micropolar fluid. Pop et al. [15] have then investigated the natural convection flow on an isothermal sphere in a micropolar fluid.

There also appear some works on the natural boundary layer flow from a sphere. For example see Chiang et al. [16], where they investigated the laminar free convection from a sphere by considering prescribed surface temperature and surface heat flux. In 1987, Huang and Chen [17] studied the natural convection from a sphere with blowing and suction. Analysis of mixed, forced and free convection about a sphere was performed by Chen and Mucoglu [18]. Very recently, Molla et al. [19] have studied the conjugate problem of heat and mass transfer from a sphere with chemical reaction.

To our best of knowledge, the effect of heat generation on a free convection flow from an isothermal circular cylinder has not been studied yet and the present work demonstrates the issue.

In the present study, it is proposed to investigate the natural convection flow of a viscous incompressible fluid from an isothermal horizontal circular cylinder considering the temperature dependent internal heat generation. The basic equations of motion are transformed into the local non-similarity boundary layer equations, which are solved numerically using a very efficient finite-difference scheme together with the Keller-box method [20, 21] and the series solution technique of Runge-Kutta-Butcher [22] together with the Nachtsheim-Swigert iteration scheme [23]. Consideration is given to the situation where the buoyancy forces assist the natural convection flow for various combinations of the heat generation parameter $\gamma$.

\section{Formulation of problem}

A steady two-dimensional laminar free convective flow from an isothermal horizontal circular cylinder of radius $a$, which is immersed in a viscous incompressible fluid, has been considered. It is assumed that the surface temperature of the cylinder is $T_{w}$, where $T_{w}>T_{\infty}$. Here $T_{\infty}$ is the ambient temperature of the fluid and $T$ is the temperature of the fluid. The physical configuration considered is as shown in Fig. 1. 


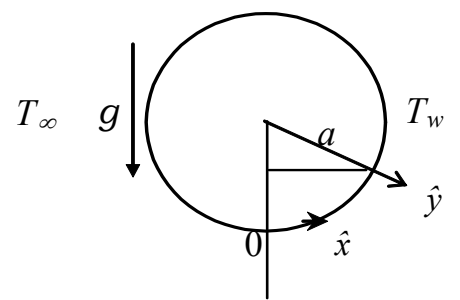

$\uparrow \uparrow \uparrow \uparrow$

Fig.1: Physical model and coordinate system.

Under the usual Bousinesq and boundary layer approximations, the equations for mass continuity, momentum and energy take the following form:

$$
\begin{aligned}
& \frac{\partial \hat{u}}{\partial \hat{x}}+\frac{\partial \hat{v}}{\partial \hat{y}}=0, \\
& \rho\left(\hat{u} \frac{\partial \hat{u}}{\partial \hat{x}}+\hat{v} \frac{\partial \hat{u}}{\partial \hat{y}}\right)=\mu \frac{\partial^{2} \hat{u}}{\partial \hat{y}^{2}}+\rho g \beta\left(T-T_{\infty}\right) \sin \left(\frac{\hat{x}}{a}\right), \\
& \hat{u} \frac{\partial T}{\partial \hat{x}}+\hat{v} \frac{\partial T}{\partial \hat{y}}=\frac{k}{\rho C_{p}} \frac{\partial^{2} T}{\partial \hat{y}^{2}}+\frac{Q_{0}}{\rho C_{p}}\left(T-T_{\infty}\right),
\end{aligned}
$$

where $(\hat{u}, \hat{v})$ are the velocity components along the $(\hat{x}, \hat{y})$ axes, $g$ is the acceleration due to gravity, $\rho$ is the fluid density, $k$ is the thermal conductivity, $\beta$ is the coefficient of thermal expansion, $\mu$ is the viscosity of the fluid, $C_{p}$ is the specific heat at constant pressure, and $Q_{0}$ is a heat generation constant which may be either positive or negative. This source term represents the heat generation when $Q_{0}>0$ and the heat absorption when $Q_{0}$ $<0$.

The appropriate boundary conditions to solve equations (1)-(3) are

$$
\begin{aligned}
& \hat{u}=\hat{v}=0, T=T_{w} \text { at } \hat{y}=0, \\
& \hat{u} \rightarrow 0, T \rightarrow T_{\infty} \text { as } \hat{y} \rightarrow \infty .
\end{aligned}
$$

To make the above equations dimensionless, we introduce the following nondimensional variables

$$
\begin{aligned}
& x=\frac{\hat{x}}{a}, y=G r^{1 / 4}\left(\frac{\hat{y}}{a}\right), \\
& u=\frac{a}{v} G r^{-1 / 2} \hat{u}, \quad v=\frac{a}{v} G r^{1 / 4} \hat{v}, \\
& \theta=\frac{T-T_{\infty}}{T_{w}-T_{\infty}}, G r=g \beta\left(T_{w}-T_{\infty}\right) \frac{a^{3}}{v^{2}},
\end{aligned}
$$

where $v(=\mu / \rho)$ is the reference kinematic viscosity, $G r$ is the Grashof number and $\theta$ is the non-dimensional temperature function.

Substituting the variables (5) into equations (1)-(3) lead to the following nondimensional equations 


$$
\begin{aligned}
& \frac{\partial u}{\partial x}+\frac{\partial v}{\partial y}=0 \\
& u \frac{\partial u}{\partial x}+v \frac{\partial u}{\partial y}=\frac{\partial^{2} u}{\partial y^{2}}+\theta \sin x \\
& u \frac{\partial \theta}{\partial x}+v \frac{\partial \theta}{\partial y}=\frac{1}{\operatorname{Pr}} \frac{\partial^{2} \theta}{\partial y^{2}}+\frac{Q_{0} a^{2}}{\mu C_{p} G r^{1 / 2}} \theta
\end{aligned}
$$

and the corresponding boundary conditions are

$$
\begin{aligned}
& u=v=0, \theta=1 \text { at } y=0, \\
& u \rightarrow 0, \theta \rightarrow 0 \text { as } y \rightarrow \infty .
\end{aligned}
$$

To solve equations (6)-(8), subject to the boundary conditions (9), we assume the following transformations

$$
\psi=x f(x, y), \quad \theta=\theta(x, y),
$$

where $\psi$ is the stream function defined in the usual way as

$$
u=\frac{\partial \psi}{\partial y}, \quad v=-\frac{\partial \psi}{\partial x} .
$$

Substituting (11) into equations (6)-(9) and after some algebraic manipulations, the transformed equations take the following form:

$$
\begin{gathered}
\frac{\partial^{3} f}{\partial y^{3}}+f \frac{\partial^{2} f}{\partial y^{2}}-\left(\frac{\partial f}{\partial y}\right)^{2}+\frac{\sin x}{x} \theta=x\left(\frac{\partial f}{\partial y} \frac{\partial^{2} f}{\partial x \partial y}-\frac{\partial f}{\partial x} \frac{\partial^{2} f}{\partial y^{2}}\right) \\
\frac{1}{\operatorname{Pr}} \frac{\partial^{2} \theta}{\partial y^{2}}+f \frac{\partial \theta}{\partial y}+\gamma \theta=x\left(\frac{\partial f}{\partial y} \frac{\partial \theta}{\partial x}-\frac{\partial \theta}{\partial y} \frac{\partial f}{\partial x}\right)
\end{gathered}
$$

where $\gamma=a^{2} Q_{0} / C_{\mathrm{p}} \mu G r^{1 / 2}$ is the heat generation parameter. The corresponding boundary conditions then turn into

$$
\begin{aligned}
& f=\frac{\partial f}{\partial y}=0, \quad \theta=1 \text { at } y=0, \\
& \frac{\partial f}{\partial y} \rightarrow 0, \quad \theta \rightarrow 0 \text { as } y \rightarrow \infty .
\end{aligned}
$$

It can be seen that near the lower stagnation point of the cylinder i.e. when $x \approx 0$, equations (12) and (13) reduce to the following ordinary differential equations

$$
\begin{aligned}
& f^{\prime \prime \prime}+f f^{\prime \prime}-f^{\prime 2}+\theta=0, \\
& \frac{1}{\operatorname{Pr}} \theta^{\prime \prime}+f \theta^{\prime}+\gamma \theta=0,
\end{aligned}
$$

which are solved using the following boundary conditions

$$
\begin{aligned}
& f=f^{\prime}=0, \theta=1, \quad \text { at } y=0, \\
& f^{\prime} \rightarrow 0, \theta \rightarrow 0 \text { as } y \rightarrow \infty .
\end{aligned}
$$

In the above equations primes denote differentiation with respect to $y$.

The physical quantities of principle interest are the shearing stress and the rate of heat transfer in terms of the skin-friction coefficient $C_{f}$ and the Nusselt number $N u$ respectively, which can be written as 


$$
\begin{aligned}
& C_{f}=\frac{\tau_{w}}{\rho U_{\infty}^{2}}, N u=\frac{a q_{w}}{k\left(T_{w}-T_{\infty}\right)} \\
& \text { where } \tau_{w}=-\mu\left(\frac{\partial \hat{u}}{\partial \hat{y}}\right)_{\hat{y}=0} \\
& \text { and } q_{w}=-k\left(\frac{\partial T}{\partial \hat{y}}\right)_{\hat{y}=0} .
\end{aligned}
$$

Using the variables (5) and the boundary condition into equation (18), we have

$$
\begin{aligned}
& C_{f} G r^{1 / 4}=x f^{\prime \prime}(x, 0), \\
& N u G r^{-1 / 4}=-\theta(x, 0) .
\end{aligned}
$$

The results of the velocity and temperature distributions are then calculated respectively from the following relations

$$
u=\frac{\partial f}{\partial y}, \quad \theta=\theta(x, y)
$$

\section{Numerical Methods}

Investigating the present problem we have employed two numerical methods, namely, the Keller box method and the perturbation solution technique, which are individually presented below.

\subsection{Implicit Finite Difference Method (FD)}

A very efficient and accurate implicit finite difference method (the Keller box method) is employed to solve the nonlinear system of partial differential equations (12)(13). The equations (12)-(13) are written in terms of first order equations in $y$, which are then expressed in finite difference form by approximating the functions and their derivatives in terms of the central differences in both coordinate directions. Denoting the mesh points in the $(x, y)$ plane by $x_{i}$ and $y_{j}$, where $i=1,2,3, \ldots, M$ and $j=1,2,3, \ldots, N$, central difference approximations are made such that the equations involving $x$ explicitly are centred at $\left(x_{i-1 / 2}, y_{j-1 / 2}\right)$ and the remainder at $\left(x_{i}, y_{j-1 / 2}\right)$, where $y_{j-1 / 2}=\left(y_{j}+y_{j-1}\right) / 2$, etc. This results in a set of nonlinear difference equations for the unknowns at $x_{i}$ in terms of their values at $x_{i-1}$. These equations are then linearised by the Newton's quasi-linearization technique and are solved using a block-tridiagonal algorithm, taking as the initial iteration of the converged solution at $x=x_{i-1}$. Now to initiate the process at $x=0$, we first provide guess profiles for all five variables (arising the reduction to the first order form) and use the Keller box method to solve the governing ordinary differential equations. Having obtained the lower stagnation point solution it is possible to march step by step along the boundary layer. For a given value of $x$, the iterative procedure is stopped when the difference in computing the velocity and the temperature in the next iteration is less than $10^{-4}$, i.e. when $\left|\delta f^{i}\right| \leq 10^{-4}$, where the superscript denotes the iteration number. The computations were not performed using a uniform grid in the $y$ direction, but a non uniform grid was used and defined by $y_{j}=\sinh ((j-1) / p)$, with $j=1,2, \ldots, 301$ and $p=100$. 


\subsection{Series solution (SS)}

Series solution of the equations (12)-(13) may be obtained by using perturbation method treating $x$ as a perturbation parameter. Here we expand the functions $f(x, y)$ and $\theta$ $(x, y)$ in powers of $x$ as

$$
\begin{aligned}
& f(x, y)=\sum_{i=0}^{\infty} x^{2 i} f_{i}(y), \\
& \theta(x, y)=\sum_{i=0}^{\infty} x^{2 i} \theta_{i}(y) .
\end{aligned}
$$

We also use the sine series

$$
\sin x=x-\frac{1}{6} x^{3}+\frac{1}{120} x^{5}-\cdots .
$$

Substituting the above expansions into equations (12)-(13) and equating the various powers of $x$ up to $O\left(x^{4}\right)$, that means for $i=0,1,2$, we get the following sets of equation

$$
\begin{gathered}
f_{0}^{\prime \prime \prime}+f_{0} f_{0}^{\prime \prime}-f_{0}^{\prime 2}+\theta_{0}=0, \\
\frac{1}{\operatorname{Pr}} \theta_{0}^{\prime \prime}+f_{0} \theta_{0}^{\prime}+\gamma \theta_{0}=0, \\
f_{0}(0)=f_{0}^{\prime}(0)=0, \quad \theta_{0}(0)=1, \\
f_{0}^{\prime}(\infty)=\theta_{0}(\infty)=0, \\
f_{1}^{\prime \prime \prime}+f_{0} f_{1}^{\prime \prime}+3 f_{0}^{\prime \prime} f_{1}-4 f_{0}^{\prime} f_{1}^{\prime}+\theta_{1}-\frac{1}{6} \theta_{0}=0, \\
\frac{1}{\operatorname{Pr}} \theta_{1}^{\prime \prime}+f_{0} \theta_{1}^{\prime}+3 \theta_{0}^{\prime} f_{1}-2 f_{0}^{\prime} \theta_{1}+\gamma \theta_{0}=0, \\
f_{1}(0)=f_{1}^{\prime}(0)=0, \quad \theta_{1}(0)=0, \\
f_{1}^{\prime}(\infty)=\theta_{1}(\infty)=0,
\end{gathered}
$$

and

$$
\begin{gathered}
f_{2}^{\prime \prime \prime}+f_{0} f_{2}^{\prime \prime}+3 f_{1}^{\prime \prime} f_{1}+5 f_{0}^{\prime \prime} f_{2}-3 f_{1}^{\prime 2}-6 f_{0}^{\prime} f_{2}^{\prime}+\theta_{2}-\frac{1}{6} \theta_{1}+\frac{1}{120} \theta_{0}=0, \\
\frac{1}{\operatorname{Pr}} \theta_{2}^{\prime \prime}+f_{0} \theta_{2}^{\prime}+3 \theta_{1}^{\prime} f_{1}+5 \theta_{0}^{\prime} f_{2}-2 f_{1}^{\prime} \theta_{1}-4 f_{0}^{\prime} \theta_{2}+\gamma \theta_{2}=0, \\
f_{2}(0)=f_{2}^{\prime}(0)=0, \quad \theta_{2}(0)=0, \\
f_{2}^{\prime}(\infty)=\theta_{2}(\infty)=0 .
\end{gathered}
$$

Here prime denotes the differentiation with respect to $y$.

The equations (24)-(32) are solved pair-wise one after another by using the Runge-Kutta-Butcher [22] initial value solver together with the Nachtsheim-Swigert [23] iteration scheme. The solutions are obtained for $f_{\mathrm{i}}$ and $\theta_{\mathrm{i}}(i=0,1,2)$ and their derivatives.

Knowing the values of $f_{\mathrm{i}}$ and $\theta_{\mathrm{i}}$ for $i=0,1,2$ and their derivatives, we can calculate the local skin-friction coefficient and the local Nusselt number from the following expressions

$$
C_{f} G r^{1 / 4}=x f^{\prime \prime}(x, 0)=x\left[f_{0}^{\prime \prime}(0)+x^{2} f_{1}^{\prime}(0)+x^{4} f_{2}^{\prime \prime}(0)\right],
$$




$$
N u G r^{-1 / 4}=-\theta^{\prime}(x, 0)=-\left[\theta_{0}^{\prime}(0)+x^{2} \theta_{1}^{\prime}(0)+x^{4} \theta_{2}^{\prime}(0)\right] .
$$

The resulting values of the local skin-friction coefficient and the local Nusselt number are depicted in Table 1 and Fig. 2. These results are compared with the corresponding values obtained from the finite difference solution.

\section{Results and discussion}

In this section we present our numerical results at various formats to understand the effects of heat generation inside the fluid flow from an isothermal circular cylinder. Initially for $\gamma=0.0$, we have reproduced some results of the local skin-friction co-efficient and the local Nusselt number of Merkin [1] in order to validate our numerical results, and later the results of the effects of heat generation are presented.

Without the effect of heat generation, i.e. for $\gamma=0.0$, a comparison of the present numerical results with those of Merkin [1] is depicted in Table 1, where we have obtained the results for the local skin-friction coefficient $C_{f} G r^{1 / 4}$ and the local Nusselt number $N u G r^{-1 / 4}$ at various positions on the surface of the circular cylinder. The fluid Prandtl number is taken to be $\mathrm{Pr}=1.0$ here. The comparison shows that the present results obtained using both the numerical techniques have an excellent agreement with the solutions of Merkin [1].

For different values of the heat generation parameter $\gamma(=0.0,0.2,0.5,0.8,1.0)$, the numerical results of the local skin-friction coefficient $C_{f} G r^{1 / 4}$ and the local Nusselt number $\mathrm{NuGr} r^{-1 / 4}$ against the curvature parameter from the lower stagnation point of the circular cylinder $(x \approx 0.0)$ to the upper stagnation point $(x \approx \pi)$ are illustrated in Figs. 2(a)-(b) respectively. The fluid Prandtl number is chosen to be $\mathrm{Pr}=0.7$. From both figures, it can be glanced how the presence of heat generation inside the fluid influences the overall predictions of $C_{f} G r^{1 / 4}$ and $N u G r^{-1 / 4}$. As the amount of heat generation increases, the local skin-friction coefficient $C_{f} G r^{1 / 4}$ increases gradually but the local Nusselt number $\mathrm{NuGr} r^{-1 / 4}$ decreases and becomes negative for a large value of $\gamma$, for example see the curves when $\gamma$ is greater than 0.2 . This is due to the fact that the heat generation mechanism creates a layer of hot fluid near the surface and at some level when $\gamma$ is large the resultant temperature of fluid finally exceeds the surface temperature (for example, see Figs $3(a)$ and $5(b)-(c))$ and that is why the rate of heat transfer from the surface decreases. This phenomenon will also be discussed elaborately latter when we present the results of the fluid velocity and temperature distributions in Figs 3(a)-(b) and the streamlines and isotherms in Figs 4 and 5.

From Figs. 2(a)-(b) we can also notice that the agreement between the results obtained by using the finite difference method and the perturbation technique (series solution) is excellent. Therefore, in Figs 3-5 we have presented the results obtained only by the finite difference method.

Figs. 3(a)-(b) illustrate the velocity and temperature distributions at $x=\pi / 6$ against $y$ for different values of the heat generation parameter $\gamma(=0.0,0.2,0.5,0.8,1.0)$ while $\mathrm{Pr}=0.7$. These figures show how the heat generation mechanism enhances the fluid velocity and temperature. Fig 3(b) clearly shows when $\gamma$ increases, the fluid temperature gradually increases and for $\gamma \geq 0.5$ it exceeds the level of surface temperature $(\theta$ 
$=1)$ and there appear to have critical levels of temperature close to the surface of the cylinder. For $\gamma=1.0$ the fluid temperature nearly doubles the surface temperature.

From Fig 3(a) we can also notice that the fluid velocity increases with $\gamma$ as this contributes to accelerate the flow and at the same time enhances the level of local skinfriction coefficient (see Fig 2(a)).

Figs. 4 and 5 illustrate the effect of the heat generation parameter $\gamma$ on the development of streamlines and isotherms, which are plotted for $\mathrm{Pr}=0.7$. From Fig. 4(a), it is seen that without the effect of heat generation (i.e. $\gamma=0.0$ ) the non-dimensional value of $\psi_{\max }$ within the computational domain is about 2.82 located at the upper stagnation point $(x \approx \pi)$ of the cylinder and when the thickness of the boundary layer reaches to the maximum level, but $\psi_{\max }$ again increases with $\gamma$ and it attains about 4.10 for $\gamma=0.5$ (see Fig 4(b)) and 5.59 for $\gamma=1.0$ (see Fig 4(c)). This phenomenon fully coincides with the early discussion made on Fig. 3(a), the fluid speeds up as $\gamma$ increases and the thickness of the velocity boundary layer grows substantially.

The isotherm patterns for corresponding values of $\gamma$ are shown in Fig. 5. From all these frames, we can see that the growth of the thermal boundary layer over the surface of the cylinder is significant. As $x$ increases from the lower stagnation point $(x \approx 0.0)$, the hot fluid raises up due to the gravity hence the thickness of the thermal boundary layer, $y$, is expected to grow. But this phenomenon is not very straightforward, as can be seen in frames 5(b) for $\gamma=0.5$ and 5(c) for $\gamma=1.0$, there appear deepness on the isotherms near to the surface of the cylinder. In both cases the levels of isotherm are noticeably higher than the surface level and the fluid temperature exceeds the level of surface temperature, which was also noticed in Fig. 3(b).

\section{Conclusion}

The effect of heat generation on natural convection flow from an isothermal horizontal circular cylinder has been investigated numerically. The governing boundary layer equations of motion are transformed into a non-dimensional form and the resulting nonlinear systems of partial differential equations are reduced to local non-similarity boundary layer equations which are solved numerically by two distinct efficient methods namely (i) Implicit finite difference method together with the Keller-box scheme and (ii) Series solution technique. We have found that for increasing values of $\gamma$ the skin-friction coefficient $C_{f} G r^{1 / 4}$ increases but the Nusselt number $N u G r^{-1 / 4}$ decreases owing to increase of the fluid temperature. With the effect of heat generation both the velocity and temperature distributions increase significantly and the thickness of the thermal boundary layer enhances.

\section{References}

[1] J.H. Merkin, Free convection boundary layer on an isothermal horizontal circular cylinders, ASME/AIChE, Heat Transfer Conference, St. Louis, Mo., August 9-11 (1976).

[2] K. Vajravelu, A. Hadjinicolaou, Heat transfer in a viscous fluid over a stretching sheet with viscous dissipation and internal heat generation, International Communication Heat Mass Transfer 20 (1993) 417-430. 
[3] A. J. Chamkha, Issa Camille, Effects of heat generation/absorption and the thermophoresis on hydromagnetic flow with heat and mass transfer over a flat plate, International Journal of Numerical Methods for Heat \& Fluid Flow 10(4) (2000) 432-448.

[4] F. Mendez, C. Trevino, The conjugate conduction-natural convection heat transfer along a thin vertical plate with non-uniform internal heat generation, International Journal of Heat Mass Transfer 43 (2000) 2739-2748.

[5] M. M. Molla, M. A. Hossain, L. S. Yao, natural convection flow along a vertical wavy surface with heat generation/absorption, International Journal of Thermal Science. 43 (2004) 157-163

[6] E.M. Sparrow, Lee L., Analysis of mixed convection about a circular cylinder, International Journal of Heat Mass Transfer, 19 (1976) 229-236.

[7] J.H. Merkin, Mixed convection a horizontal circular cylinder, International Journal of Heat Mass Transfer, 20 (1977) 73-77.

[8] J.H. Merkin, Free convection boundary layer on cylinders of elliptic cross-section, ASME, Journal of Heat Transfer, 99 (1977) 453-457.

[9] D.B. Ingham, Free convection boundary layer on an isothermal horizontal cylinder, Z. Angew. Math. Phys., 29 (1978) 871-883.

[10] M.A. Hossain, M.A. Alim, Natural convection- radiation interaction on boundary layer flow along a vertical thin cylinder, Heat and Mass Transfer, 32 (1997) 515-520.

[11] M.A. Hossain, M. Kutubuddin, I. Pop, Radiation-conduction interaction on mixed convection a horizontal circular cylinder, Heat and Mass Transfer, 35 (1999) 307314.

[12] M. M. Molla, M. A. Hossain, R. S. R. Gorla, Natural convection flow from an isothermal horizontal circular cylinder with temperature dependent viscosity, Heat and Mass Transfer, 41 (2005) 594-598.

[13]I. Pop, R. Nazar, N. Amin, Free convection boundary layer on an isothermal horizontal circular cylinder in a micropolar fluid, Heat Transfer, Proceeding of the Twelfth International Conference (2002).

[14], I. Pop, R. Nazar, N. Amin, Free convection boundary layer on a horizontal circular cylinder wit constant heat flux in a micropolar fluid, International Journal Applied Mechanics \& Engineering 7 (2) (2002) 409-431.

[15]I. Pop, T. Grosan, N. Amin, R. Nazar, Free convection boundary layer on an isothermal sphere in a micropolar fluid, International Communication Heat Mass Transfer 29 (3) (2002) 377-386.

[16] T. Chiang, A. Ossin, C. L. Tien, Laminar free convection from a sphere, ASME Journal Heat Transfer 86 (1964) 537-542.

[17] M. J. Huang, C. K. Chen, Laminar free convection from a sphere with blowing and suction, ASME Journal of Heat Transfer 109 (1987) 529-532.

[18] T.S. Chen, A. Mocoglu, Analysis of mixed forced and free convection about a sphere, International Journal of Heat Mass Transfer 20 (1977) 867-875.

[19] M. M. Molla, M. A. Hossain, R. S. R. Gorla, Conjugate effect heat and mass transfer in natural convection flow from an isothermal sphere with chemical reaction, International Journal of Fluid Mechanics Research 31(4) (2004) 319-331.

[20] T. Cebeci, P. Bradshaw, Physical and Computational Aspects of Convective heat Transfer, Springer, New York 1984.

[21]H.B. Keller, Numerical methods in boundary layer theory, Annual Review Fluid Mechanics 10 (1978) 417-433. 
[22] J.C. Butcher, Implicit Runge-Kutta process, Math Comp. 18 (1974) 50-55.

[23] P. R. Nachtsheim, P. Swigert, Satisfaction of Asymptotic Boundary Conditions in Numerical Solutions Systems of Non-linear Equations of Boundary layer Type, NASA TN-D3004 (1965). 
Table 1: Comparisons of the present numerical results with those of Merkin [1] for $\mathrm{Pr}=$ 1.0 and $\gamma=0$.

\begin{tabular}{ccccccc}
\hline & \multicolumn{3}{c}{$C_{f} G r^{1 / 4}$} & & $N u G r^{-1 / 4}$ & \\
\hline$x$ & $\begin{array}{c}\text { Merkin } \\
{[1]}\end{array}$ & $\begin{array}{c}\text { Finite diff. } \\
\text { solutions }\end{array}$ & $\begin{array}{c}\text { Series } \\
\text { solutions }\end{array}$ & $\begin{array}{c}\text { Merkin } \\
{[1]}\end{array}$ & $\begin{array}{c}\text { Finite diff. } \\
\text { solutions }\end{array}$ & $\begin{array}{c}\text { Series so- } \\
\text { lutions }\end{array}$ \\
\hline 0.0 & 0.0000 & 0.0000 & 0.0000 & 0.4214 & 0.4241 & 0.4216 \\
$\pi / 6$ & 0.4151 & 0.4145 & 0.4144 & 0.4161 & 0.4161 & 0.4164 \\
$\pi / 3$ & 0.7558 & 0.7539 & 0.7544 & 0.4007 & 0.4005 & 0.4009 \\
$\pi / 2$ & 0.9579 & 0.9541 & 0.9550 & 0.3745 & 0.3740 & 0.3751 \\
$2 \pi / 3$ & 0.9756 & 0.9696 & 0.9701 & 0.3364 & 0.3355 & 0.3389 \\
$5 \pi / 6$ & 0.7822 & 0.7739 & 0.7824 & 0.2825 & 0.2812 & 0.2923 \\
$\pi$ & 0.3391 & 0.3264 & 0.4125 & 0.1945 & 0.1917 & 0.2354 \\
\hline
\end{tabular}



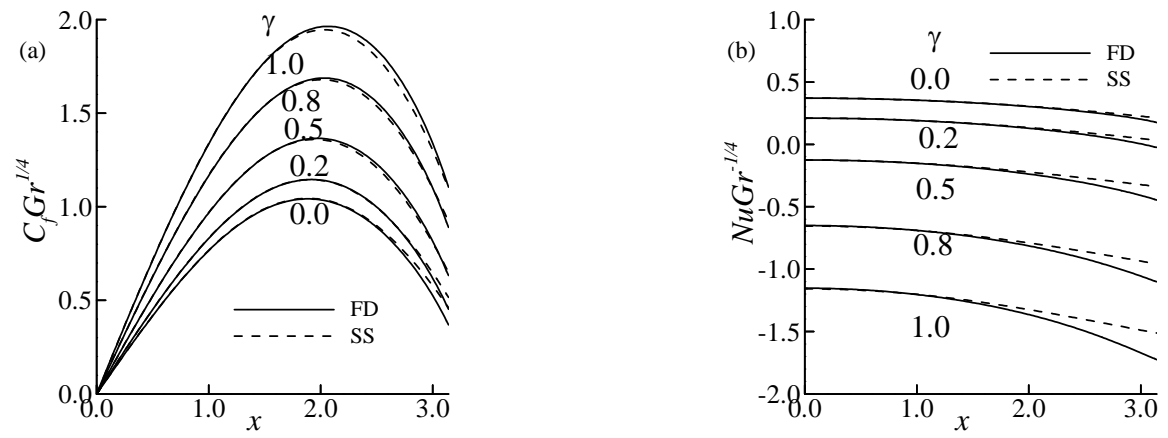

Fig. 2: (a) Skin-friction coefficient and (b) Rate of heat transfer for different values of $\gamma$ while $\operatorname{Pr}=0.7$. 

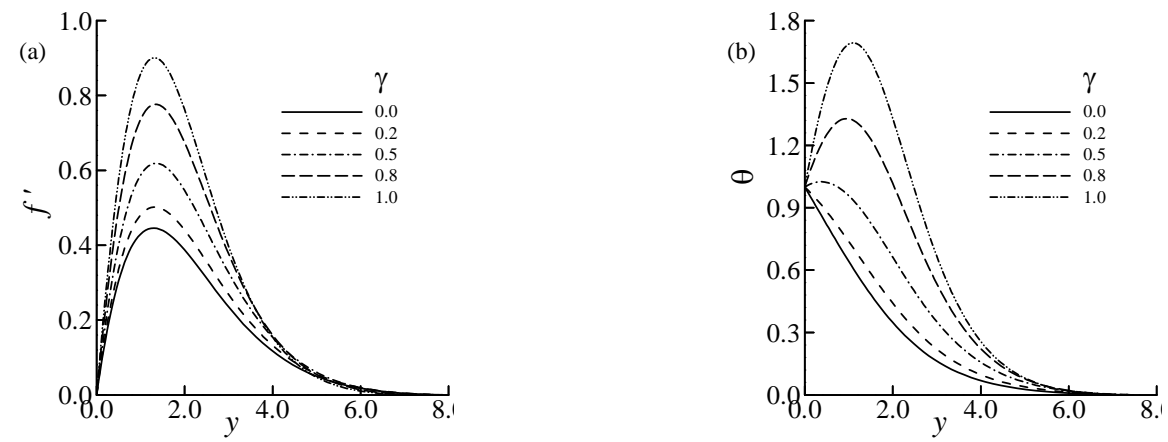

Fig. 3: (a) Velocity and (b) Temperature distributions for different values of $\gamma$ while $\operatorname{Pr}=$ 0.7 . 

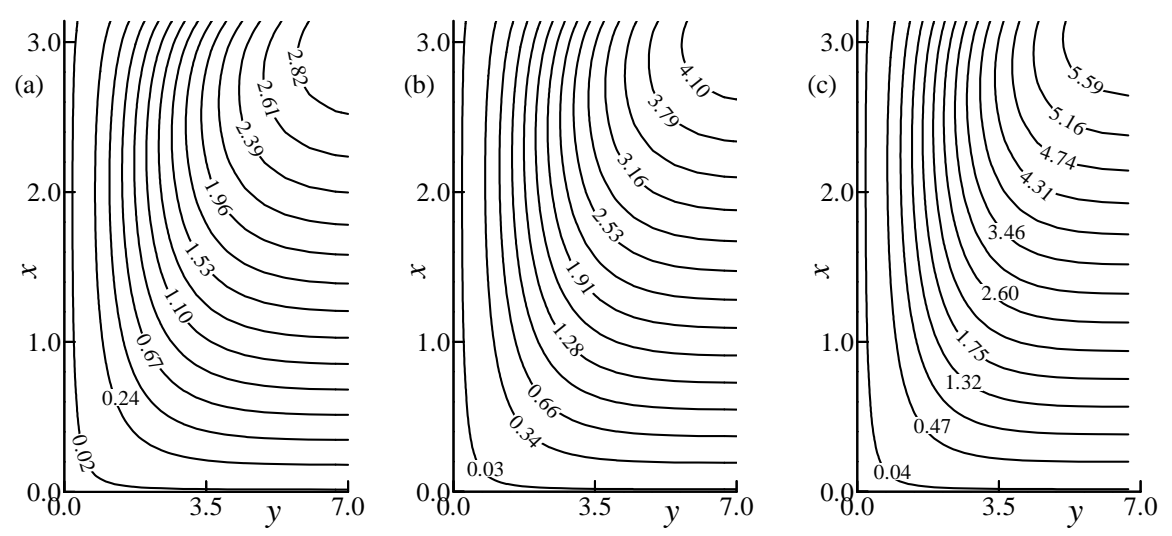

Fig. 4: Streamlines for (a) $\gamma=0.0$ (b) $\gamma=0.5$ and (c) $\gamma=1.0$ while $\operatorname{Pr}=0.7$. 

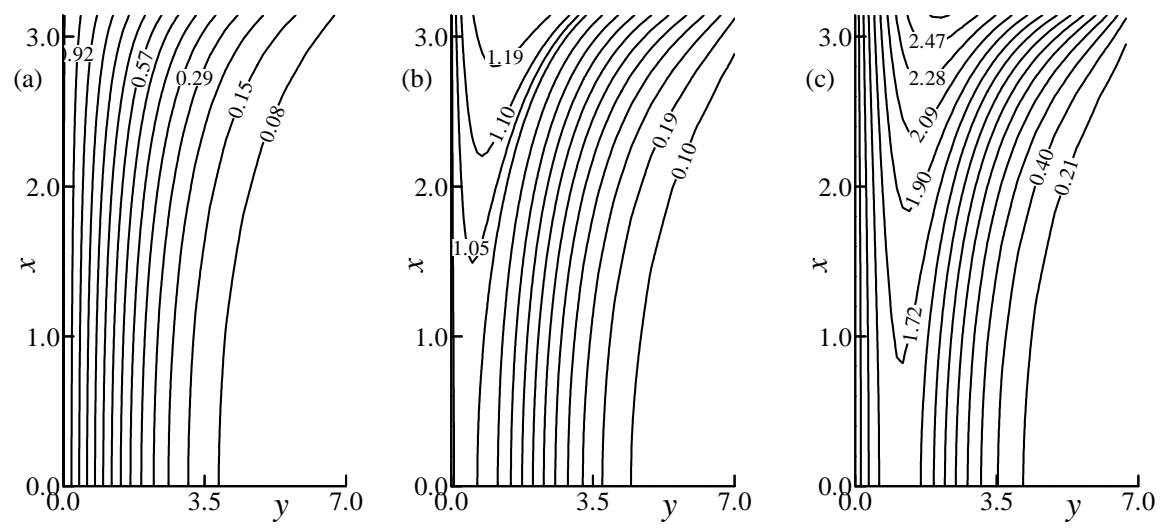

Fig. 5: Isotherms for (a) $\gamma=0.0$ (b) $\gamma=0.5$ and (c) $\gamma=1.0$ while $\operatorname{Pr}=0.7$. 